Research Article

\title{
Seasonal Profile and Five-Year Trend Analysis of Malaria Prevalence in Maygaba Health Center, Welkait District, Northwest Ethiopia
}

\author{
Fitsum Tigu $\mathbb{D},{ }^{1}$ Tsegay Gebremaryam, ${ }^{2}$ and Asnake Desalegn $\mathbb{D}^{1}$ \\ ${ }^{1}$ Department of Microbial, Cellular and Molecular Biology, Division of Microbiology, College of Natural Sciences, \\ Addis Ababa University, Addis Ababa, Ethiopia \\ ${ }^{2}$ Department of Zoological Sciences, College of Natural Sciences, Addis Ababa University, Addis Ababa, Ethiopia
}

Correspondence should be addressed to Fitsum Tigu; fitsum.tigu@aau.edu.et

Received 19 April 2021; Revised 26 August 2021; Accepted 1 September 2021; Published 11 September 2021

Academic Editor: Lizandra Guidi Magalhães

Copyright (C) 2021 Fitsum Tigu et al. This is an open access article distributed under the Creative Commons Attribution License, which permits unrestricted use, distribution, and reproduction in any medium, provided the original work is properly cited.

\begin{abstract}
Background. Malaria is a serious public health problem of most developing countries, including Ethiopia. The burden of malaria is severely affecting the economy and lives of people, particularly among the productive ages of rural society. Thus, this study was targeted to analyze the past five-year retrospective malaria data among the rural setting of Maygaba town, Welkait district, northwest Ethiopia. Methods. The study was done on 36,219 outpatients attending for malaria diagnosis during January 2015 to 2019. Data was extracted from the outpatient medical database. Chi-square $\left(\chi^{2}\right)$ test and binary logistic regression model were used to analyze the retrospective data. Statistical significance was defined at $p<0.05$. Results. Of 36,219 outpatients examined, 7,309 (20.2\%) malaria-positive cases were reported during 2015-2019. There was a fluctuating trend in the number of malaria-suspected and -confirmed cases in each year. Male slide-confirmed $(61.4 \%, N=4,485)$ were significantly higher than females $(38.6 \%, N=2,824)(p<005)$. Plasmodium falciparum and Plasmodium vivax were the dominant parasites detected, which accounted for 66.1\%; $N=4832,33.9 \% ; N=2477$, respectively. Despite the seasonal abundance of malaria cases, the highest prevalence was recorded in autumn (September to November) in the study area. Binary logistic regression analysis revealed that statistically significant associations were observed between sexes, interseasons, mean seasonal rainfall, and mean seasonal temperature with the prevalence of $P$. vivax. However, $P$. falciparum has shown a significant association with interseasons and mean seasonal temperature. Conclusions. Although the overall prevalence of malaria was continually declined from 2015-2019, malaria remains the major public health problem in the study area. The severe species of $P$. falciparum was found to be the dominant parasite reported in the study area. A collaborative action between the national malaria control program and its partners towards the transmission, prevention, and control of the two deadly species is highly recommended.
\end{abstract}

\section{Introduction}

Malaria is one of the major tropical diseases that adversely affect the public health and the economic development of many developing countries [1]. There are five known species of Plasmodium (P. falciparum, P. vivax, P. malariae, P. ovale, and $P$. knowlesi) which are able to cause malaria in humans through the bite of female anopheles mosquitoes [2]. Among which, $P$. falciparum and $P$. vivax are the most deadly para- sites that cause severe morbidity and mortality in most malarious regions of the world including Ethiopia $[2,3]$.

Malaria causes serious systemic complications in humans, including infectious in the nervous, respiratory, renal, and hematopoietic systems [4]. Particularly, the severity of $P$. falciparum malaria is much more complex than any other known species. The clinical complications include, but not limited to impaired consciousness, acidosis, hypoglycaemia, severe anaemia, renal impairment, jaundice, pulmonary 
oedema, significant bleeding, shock, and hyperparasitaemia [5]. Delayed treatment and lack of immediate supportive care may result in death.

Looking at the world malaria cases from 2010 to 2018, there was a marked decline with the exception of WHO African region. This region still carries the highest share of global malaria burden [6]. The root cause of malaria in this region is always associated with lower socioeconomic status, food insecurity, poor housing, and lack of medical care [7]. Despite the fact that malaria is associated with poverty, it is also strongly associated with environmental conditions [8]. The presence of conducive mosquito breeding sites and suitable temperature for the parasite as well as for the vector developments was frequently reported in the tropical regions of Africa [9].

In Ethiopia, the distribution and transmission of malaria are largely unstable and seasonal and dependent on altitude and climate. In addition, the majority of the people are exposed to malaria due to the presence favorable topography and climate for malaria transmission [9-11]. Ethiopia has recorded an estimated 2,927,266 new malaria cases and 4,782 deaths with a crude death rate of 4.7 per 100,000 in 2016 [12].

However, in recent decades, Ethiopia has achieved notable progress in fighting against malaria and significantly reduced malaria-related morbidity and mortality. This was achieved through coordinated efforts of the National Malaria Control and Elimination Program (NMCEP) and its partners [13]. It was tackled by intensive use of insecticide residual spray (IRS), long-lasting insecticide-treated mosquito nets (LLNs), chemotherapies, improved diagnosis, and case management and provision of quality laboratory facilities to the patients [14].

The feasibility of malaria elimination in Ethiopia and making a malaria-free Ethiopia requires concurrent investments in the high burden areas in the west, and datainformed decision-making is crucial to these efforts [13, 14]. Therefore, the aim of this study was to assess the prevalence and trend of malaria among the rural setting of Maygaba town, Welkait district, northwest Ethiopia from 2015 to 2019. Additionally, this study will give evidence-based information about the current status of malaria to all stakeholders and provide useful data for decision makers, policy makers, and malaria prevention and control strategists for the design and implementation of possible solutions.

\section{Methods}

2.1. Study Design and Setting. The study was designed to analyze a five-year (2015-2019) retrospective data extracted from Maygaba Health Center (MHC). The study was conducted in Maygaba town of Welkait district, northwest Ethiopia. The town is located about $929 \mathrm{~km}$ from the capital city, Addis Ababa, and $273 \mathrm{~km}$ from Gondar city. It has one health center and six health posts. According to the Myagaba town communication office, the total population of the study area is about 30,974, of which 15,642 are males and 15,332 are females. The altitude of the study area ranges between 677 and 2,755 meters above sea levels. The mean annual temperature is $22.5^{\circ} \mathrm{C}$ with minimum and maximum temperature ranges of 15 and $30^{\circ} \mathrm{C}$, respectively. The town and its surrounding rural areas had a total of six villages, one urban and five rural villages with 7,039 households. Each village had an average family size of 4.4 persons per household.

2.2. Data Collection. For the past five years (2015-2019), malaria health examination record data were collected from $\mathrm{MHC}$ using the format developed by the principal investigator. The format includes malaria diagnosis results (negative or positive), types of infecting Plasmodium species (for positives), year of diagnoses, and sociodemographic data. However, due to the incompleteness of the medical record sheets, the sociodemographic data were excluded from the analysis except the sex. The malaria trends in the study area were analyzed by appropriate software.

2.3. Data Analysis. Wholeness and consistency of the data were checked twice and entered into SPSS version 20 software (SPSS Inc., Chicago, IL, USA) for statistical analysis. Descriptive statistics (frequencies and percentages) were used to describe the malaria cases with respect to months, years, sex, and Plasmodia species. Chi-square $\left(\chi^{2}\right)$ test and binary logistic regression model were applied to analyze the association of the different variables with confirmed malaria cases. Statistical significance was defined at $p$ values $<0.05$.

\section{Results}

3.1. Trends of Malaria Cases in MHC. During January 2015 to December 2019, about 36,219 patients have been diagnosed for malaria and out of which $20.2 \%(N=7,309)$ were slide-positive. On average, 7,244 malaria-suspected and 1,462 malaria-confirmed cases were tested by MHC every year. Variation in the number of malaria-suspected and -confirmed cases in each year was observed. Despite the variation, the average monthly malaria prevalence was $1.7 \%$. Generally, the overall prevalence of malaria showed a declining trend during 2015 to 2019, except a slight increase recorded in 2016 (Figure 1 and Table 1).

3.2. Distribution of Confirmed Malaria Cases by Sex. Regarding the distribution of sex, from the total tested cases, slightly over half $(54.7 \%, N=19,797)$ of patients were males and $(45.3 \%, N=16,422)$ were females. While from the total slide-confirmed cases, the majority $(61.4 \%$, $N=4,485)$ of them were males and the rest $(38.6 \%, N=$ 2,824 ) were females.

Looking at the overall male to female ratio of tested and slide-confirmed cases, mostly males were affected more frequently than females with a ratio of $1.2: 1$ and $1: 0.6$, respectively. This difference was statistically significant $\left(\chi^{2}=3.923\right.$, $p<0.05$ ). Consequently each year, a higher number of malaria-positive males were observed than the malariapositive females; however, yearly difference in the number of cases was not statistically significant $(p>0.05)$. Comparing the annual overall prevalence of malaria under each sex category, males showed a higher $(22.7 \%)$ prevalence than females (17.2\%) (Table 2). 


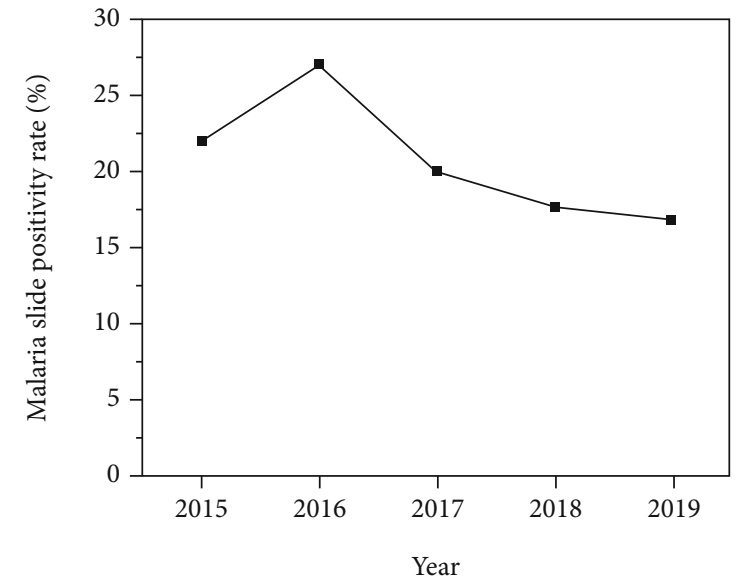

Figure 1: Trends of malaria prevalence at MHC, northwest Ethiopia, from 2015-2019.

TABLE 1: Annual trends of total malaria cases in MHC, from 20152019.

\begin{tabular}{ccc}
\hline Year & Blood films examined & Slide-confirmed malaria cases (\%) \\
\hline 2015 & 4642 & $1021(22.0)$ \\
2016 & 6153 & $1667(27.1)$ \\
2017 & 8850 & $1764(19.9)$ \\
2018 & 7255 & $1281(17.7)$ \\
2019 & 9319 & $1576(16.9)$ \\
Total & 36219 & $7309(20.2)$ \\
\hline
\end{tabular}

3.3. Seasonal Profile of Malaria Prevalence. The total number of malaria examined over malaria positive cases showed great variation between seasons. With respect to the number of cases, total cases exhibited the following order: autumn $>$ winter $>$ summer $>$ spring (Table 3 and Figure 2). Majority of suspected as well as infected cases were observed soon after the main rainy season (September to November). A total of $33.3 \%$ of malaria negative and $37.5 \%$ malaria positive cases were reported in this season (Figure 2 and Table 3). While the smallest number of malaria-suspected (17.8\%) and -infected (11.7\%) cases were reported during the small rainy season (March-May). Almost the same malaria-suspected cases (25.5 and $23.8 \%$ ) were observed in dry and heavy rainy seasons, respectively (Figure 2 and Table 3 ). A significantly higher number of slide-confirmed malaria cases $(37.5 \%)$ were observed during autumn soon after the heavy rainy season $(p<0.01)$ compared to dry and small rainy seasons.

Generally, pairwise comparisons indicate that there are significant interseasonal variations $(p<0.001)$ except between autumn and summer $(p=0.376)$. Since the actual season of malaria in the study area was not clearly defined, it is not possible to calculate the prevalence of malaria for each season. Nevertheless, this imperative result tells us that malaria was observed throughout all the seasons.

3.4. Plasmodium Species Distribution by Years and Seasons. In terms of infectious category, P. falciparum was the major $(66.1 \%)$ contributor to malaria infection in the study area, while P. vivax accounted only (33.9\%) of infection (Table 3). The difference was statistically significant $\left(\chi^{2}=758.8, p<0.001\right)$. Coinfection of $P$. falciparum and $P$. vivax was not reported at all during the 2015-2019 retrospective study. The overall trend of both infections showed variation between years and seasons. Apparently, P. falciparum was the dominant infection that occurred consistently over $66 \%$ in all study years and seasons (Figure 3 and Table 3). So, it is not surprising that $P$. falciparum was the most prevalent infection than $P$. vivax in the past five years at the study site.

Despite dominance and prevalence, both infections followed similar patterns towards seasonality (Figure 2 and Table 3). About 37.8, 31.2, and 19.1 of P. falciparum infection were registered in September to November, December to February, and June to August, respectively. Similarly, about 36.9, 31.8, and 20.3 of $P$. vivax cases were also recorded in September to November, December to February, and June to August, respectively (Figure 2 and Table 3). Although a lower number of $P$. falciparum (12\%) as well as $P$. vivax $(11 \%)$ cases was reported in the small rainy season than any other seasons, however, statistically significant difference was not noticed $\left(\chi^{2}=5.67, p=0.234\right)$.

3.5. Correlation between Malaria Type and Other Predictor Variables. The relationship between malaria type and other predictor variables (sex, season, rainfall, and temperature) was analyzed by a binary logistic regression model as indicated in Table 4. It showed that the inert-seasons and mean seasonal temperature have statistically significant $(p<0.001)$ associated with the prevalence of $P$. falciparum. Other independent factors such as sex and mean seasonal rainfall have no significance associated with it. However, statistically significant $(p<0.001)$ associations were observed between sexes, interseasons, mean seasonal rainfall, and mean seasonal temperature with the prevalence of $P$. vivax. In addition to this, significantly higher numbers of $P$. vivax cases were observed during autumn and winter seasons compared to that of summer and spring seasons. Also, significant differences were observed between males and females, where males were more affected by $P$. vivax compared to females $(\mathrm{AOR}=0.754,95 \% \mathrm{CI}=0.681-0.835, p<0.001)$.

\section{Discussion}

Malaria is the major public health concern in Ethiopia and causes significant morbidities and mortalities to the people. Particularly, the rural societies are extremely affected by this vector borne disease. Thus, the purpose of this trend analysis was to investigate the current status and impact of malaria on the rural settings across the years and seasons from 2015 to 2019. Ultimately, the findings of this research will pave the way to the creation of malaria-free-nation in Ethiopia.

The five-year retrospective data analysis revealed that the overall prevalence of malaria was showing a declining trend except a slight increase observed in the year of 2016, and this was due to the occurrence of unseasonal rainfall in the study area as the information obtained from the MHC indicates but significant association was not observed with yearly 
TABLE 2: Distribution of confirmed malaria cases by sex at MHC, from 2015-2019.

\begin{tabular}{lccccc}
\hline Sex & Total cases & Slide positive $(n, \%)$ & $P$. falciparum $(n, \%)$ & $P$. vivax $(n, \%)$ & $\chi^{2}$ \\
\hline Male & 19797 & $4485(61.4)$ & $3010(67.1)$ & $1475(32.9)$ & \\
Female & 16422 & $2824(38.6)$ & $1822(64.5)$ & $1002(35.5)$ & 3.923 \\
Total & 36219 & 7309 & $4832(66.1)$ & $2477(33.9)$ & 0.04 \\
\hline
\end{tabular}

TABLe 3: Seasonal profile and prevalence of malaria at MHC during 2015-2019.

\begin{tabular}{|c|c|c|c|c|c|}
\hline Season & Total cases & Total positive (\%) & Total negative (\%) & P. falciparum (\%) & P. $\operatorname{vivax}(\%)$ \\
\hline Summer (Jun. to Aug.) & 8615 & $1424(19.5)$ & $7191(24.9)$ & $921(19.1)$ & $503(20.3)$ \\
\hline Autumn (Sep. to Nov.) & 12371 & $2739(37.5)$ & $9632(33.3)$ & $1825(37.8)$ & $914(36.9)$ \\
\hline Winter (Dec. to Feb.) & 9224 & $2293(31.4)$ & $6931(24.0)$ & $1506(31.2)$ & $787(31.8)$ \\
\hline Spring (Mar. to May) & 6009 & $853(11.7)$ & $5156(17.8)$ & $580(12.0)$ & $273(11.0)$ \\
\hline Total & 36219 & 7309 & 28910 & $4832(66.1)$ & $2477(33.9)$ \\
\hline
\end{tabular}

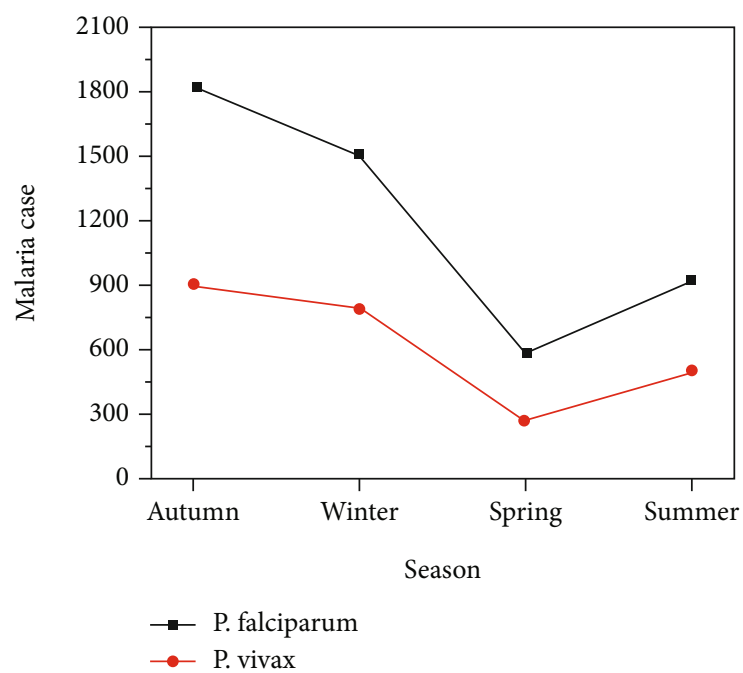

FIgURe 2: Seasonal prevalence of malaria by Plasmodium types at MHC, northwest Ethiopia, from 2015-2019.

mean rainfall data (data not shown). This might be because the rainfall data used for analysis was obtained from nearby city (Gondar), which is far by $273 \mathrm{~km}$ from the study site due to the lack of meteorological data in the exact study site.

A total of $7309(20.2 \%)$ of slide-confirmed malaria cases were recorded during the five-year study period. This finding was comparable with the study conducted in the East Wollega, $20.0 \%$ [15] and the Northwest Gondar, 21.8\% of Ethiopia [16]. However, the presence of parasitemia in this study was quite lower than similar studies conducted in various regions of Ethiopia ranged from 32.6 to $41.5 \%$ [17-20] and higher than the findings in the North Shoa, South Wello, and West Gojam Amhara region and North West Tigray of Ethiopia it ranged from 5.0 to $16.3 \%$ [21-26]. The possible reasons for the differences may be due to variations of the study settings, time of the investigation, LLN coverages, and the knowledge levels of the societies and interventions of malaria prevention and control measures.

The health record data of this study indicated that a significant number of male malaria-confirmed cases were

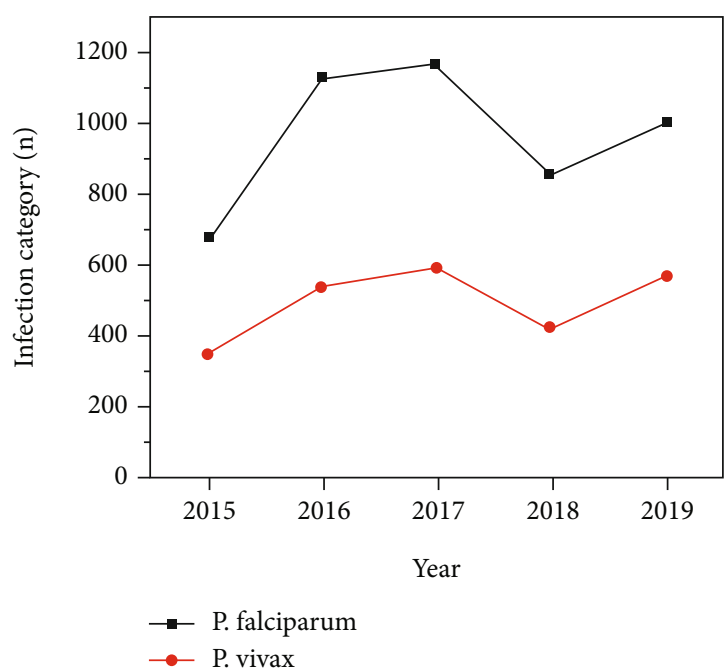

FIGURE 3: Side positive cases by infection category at MHC, northwest Ethiopia, from 2015-2019.

recorded in the MHC compared to the female, suggesting that males were extremely infected by malaria in the study area. This finding was comparable with a similar study reported in another malaria-endemic area of Ethiopia [27]. This is because males' had a greater occupational risk of getting the disease than women. Males, mainly 18-40 years old, are usually engaged in outdoor activity such as farming particularly, irrigation activity was mostly done during evening up to night in the study area. However, logistic regression analysis conducted between malaria type and sexes indicated that only $P$. vivax has a significant correlation $(\mathrm{AOR}=0.754$; $95 \% \mathrm{CI}=0.681-0.835 ; p<0.001)$ with sexes.

Malaria transmission was recorded throughout the seasons in the study area, and the interseasonal variations were significant $(p<0.001)$. The substantial number of malariainfected cases was reported shortly after the main rainy season. This is because the existence of cold and cloudy weather conditions during this season creates a conducive environment for breeding of mosquitoes [28]. Furthermore, most cultivated crops including maize release pollen grains 
TABLE 4: Binary logistic regression analysis of factors associated with $P$. falciparum and P. vivax infection during 2015-2019.

(a) With Plasmodium falciparum

\begin{tabular}{lccc}
\hline Variable & AOR & $95 \%$ CI & $p$ value \\
\hline Gender & 1 & & \\
Male & 1.074 & $0.72-1.188$ & 0.163 \\
Female & & & \\
Season & 1 & & \\
Summer (Jun. to Aug.) & 2.557 & $1.403-2.770$ & $<0.001$ \\
Autumn (Sep. to Nov.) & 1.528 & $1.422-1.661$ & $<0.001$ \\
Winter (Dec. to Feb.) & 0.581 & $0.461-0.731$ & $<0.001$ \\
Spring (Mar. to May) & 0.980 & $0.936-1.025$ & 0.379 \\
Mean seasonal rainfall & 0.788 & $0.744-0.836$ & $<0.001$ \\
Mean seasonal temperature & & &
\end{tabular}

(b) With Plasmodium vivax

\begin{tabular}{lccc}
\hline Variable & AOR & $95 \%$ CI & $p$ value \\
\hline Gender & 1 & & \\
Male & 0.754 & $0.681-0.835$ & $<0.001$ \\
Female & & & \\
Season & 1 & & \\
Summer (Jun. to Aug.) & 2.129 & $1.698-2.669$ & $<0.001$ \\
Autumn (Sep. to Nov.) & 1.966 & $1.556-2.484$ & $<0.001$ \\
Winter (Dec. to Feb.) & 0.731 & $0.651-0.995$ & $<0.001$ \\
Spring (Mar. to May) & 1.051 & $1.003-1.101$ & 0.038 \\
Mean seasonal rainfall & 1.297 & $1.229-1.369$ & $<0.001$ \\
Mean seasonal temperature & & & \\
\hline
\end{tabular}

following the rainy season may serve as a food source for mosquito larvae to complete its life cycle.

In this study, $P$. falciparum was predominantly found in the study area and became a major (66.1\%) contributor of morbidity and mortality followed by $P$. vivax. This was in agreement with the national report $[29,30]$ as well as reports from most other regions of Ethiopia [3,31,32], but in contrast to the closest region of Africa, Eastern Sudan [33]. This might be due to the fact that the pathogenesis of $P$. falciparum is stronger than the $P$. vivax $[34,35]$.

The prevalence of malaria in Ethiopia largely depends on seasons [11], and the highest transmission of both parasites ( $P$. falciparum and P. vivax) is also recorded biannually following the two rainy seasons (September to November and March to May) [27]. The coincidence of malaria transmission patterns with the peak agricultural activities significantly affects the economy of the country [32]. However, in this study, the lowest prevalence was reported in the small rainy season (March to May). This finding further strengthens the variabilities of malaria transmission in Ethiopia [36, 37].

Furthermore, binary logistic regression analysis showed that the parasite prevalence of $P$. falciparum and P. vivax has strongly correlated with meteorological data such as mean seasonal temperature and mean seasonal rainfall except the latter was not significantly associated with the prevalence of $P$. falciparum. This might be due to the fact that temperature, rainfall, and relative humidity are the major climatic factors that have an impact on malaria transmission [15]. However, the absence of a positive correlation of $P$. falciparum with rainfall in this study may be due to variable effects of the rainfall in hot areas and cold areas. The effect of rainfall in hot areas towards the prevalence of $P$. falciparum was relatively weaker than the cold areas as reported [38]. This study was in agreement with the tenyear retrospective study conducted in Western Ethiopia [15].

In the epidemiological point of view, rainfall has played an important role in development and reproduction of adult mosquitoes [39]; however, the impact of rainfall on the transmission of malaria is very complicated since the effect is dependent on various situations such as geographical region and specific to breeding habitats of mosquitoes. A study conducted by McMichael [39] indicated that moderate rains may have beneficial to mosquito breeding while heavy rains may clear out the breeding sites as well as the developing mosquito larvae.

Finally, it is difficult to draw better and reliable conclusions from five years of health recorded data, and analyzing as much recorded data as possible may provide more qualified conclusions. However, due to poor recordkeeping at MHC and lack of malaria-related follow-up data, for instance, age, morbidity, and mortality data were missing and limited our study. Furthermore, the meteorological data used in this study for binary regression analysis was obtained from nearby city, Gondar, due to a lack of data in the exact study site.

\section{Conclusions}

Although the overall prevalence of malaria was declined during the study period of 2015 to 2019, malaria remains the major public health problem in the study area. The severe species of $P$. falciparum was found to be the dominant parasite reported during the study periods. Autumn (September to November) was identified as the highest malaria prevalence season, and males are more affected than females in the study area. A collaborative action between the national malaria control program and its partners towards the transmission, prevention, and control of the two deadly species is highly recommended.

\section{Abbreviations}

MHC: $\quad$ Maygaba Health Center

LLNs: Long-lasting insecticide-treated mosquito nets

CNCS-IRB: College of Natural and Computational Sciences, Institutional Review Board

NMAE: The National Meteorological Agency of Ethiopia.

\section{Data Availability}

All relevant data are within the paper. 


\section{Ethical Approval}

The study was ethically approved by the Health Bureau of Welkait district and College of Natural Science, Institutional Review Board, Addis Ababa University (Ref. No. CNSDO/185/12/19).

\section{Consent}

Since this data was extracted from health record sheets of the patients, the "informed consent" is not applicable.

\section{Conflicts of Interest}

The authors declare that they have no competing interests.

\section{Authors' Contributions}

All authors contributed to the study conception and design. Material preparation and data collection were performed by FT and TG. Statistical analysis and interpretation were performed by FT and AD. FT wrote the main manuscript text and prepared all the figures. All authors reviewed the manuscript.

\section{Acknowledgments}

We gratefully acknowledge Addis Ababa University, School of Graduate Program for the provision of financial support. The authors also would like to thank the College of Natural and Computational Sciences, Institutional Review Board (CNS-IRB) for ethical approval. We are also thankful to the Federal Minister of Education, School of Biological Sciences and Biotechnology, MHC and laboratory technicians. Finally, we would like to thank the National Meteorological Agency of Ethiopia (NMAE) for the provision of meteorological data of Gondar from 2015-2019. The research was funded by Addis Ababa University for data collection.

\section{References}

[1] A. A. Goodman, "Malaria: The King of Tropical Diseases," vol. 259, 2016https://www.thegreatcoursesdaily.com/malariaking-tropical-diseases/.

[2] WHO, "Malaria," 2019, https://www.who.int/health-topics/ malaria\#tab=tab_1.

[3] H. S. Taffese, E. Hemming-Schroeder, C. Koepfli et al., "Malaria epidemiology and interventions in Ethiopia from 2001 to 2016," Infectious Diseases of Poverty, vol. 7, no. 1, p. 103, 2018.

[4] A. Trampuz, M. Jereb, I. Muzlovic, and R. M. Prabhu, "Clinical review: severe malaria," Critical Care, vol. 7, no. 4, pp. 315$319,2003$.

[5] J. Wiley, "Severe malaria," Tropical Medicine \& International Health, vol. 19, supplement 1, pp. 7-131, 2014.

[6] WHO, World malaria report, World Health Organization, Geneva, Switzerland, 2019.

[7] L. S. Tusting, J. Rek, E. Arinaitwe et al., "Why is malaria associated with poverty? Findings from a cohort study in rural Uganda," Infectious Diseases of Poverty, vol. 5, no. 1, p. 78, 2016.
[8] A. Rossati, O. Bargiacchi, V. Kroumova, M. Zaramella, A. Caputo, and P. L. Garavelli, "Climate, environment and transmission of malaria," Le Infezioni in Medicina, vol. 24, no. 2, pp. 93-104, 2016.

[9] G. Zhou, N. Minakawa, A. K. Githeko, and G. Yan, "Association between climate variability and malaria epidemics in the East African highlands," Proceedings of the National Academy of Sciences, vol. 101, no. 8, pp. 2375-2380, 2004.

[10] CDKN, "FEATURE: Climate information to help combat malaria in Ethiopia," 2019, https://cdkn.org/2019/04/featureclimate-information-to-help-combat-malaria-in-ethiopia/ ?loclang=en_gb/.

[11] B. B. Dadi, "Modeling Malaria Cases Associated with Environmental Risk Factors in Ethiopia Using Geographically Weighted Regression," 2020.

[12] T. Girum, T. Shumbej, and M. Shewangizaw, "Burden of malaria in Ethiopia, 2000-2016: findings from the Global Health Estimates 2016," Tropical Diseases, Travel Medicine and Vaccines, vol. 5, no. 1, p. 11, 2019.

[13] Charting the path to malaria elimination, PATH, Ethiopia, 2019, https://path.azureedge.net/media/documents/EthiopiaImpact-Report.pdf.

[14] Gessessew Bugssa and Kiros Tedla, "Feasibility of malaria elimination in Ethiopia," Ethiopian Journal of Health Sciences, vol. 30, no. 4, pp. 607-614, 2020.

[15] T. Gemechu, A. Samuel, and D. Yewhalaw, "Ten years trend analysis of malaria prevalence and its correlation with climatic variables in Sibu Sire District, east Wollega zone, Oromia regional state, Western Ethiopia: a retrospective study," Science, Technology and Arts Research Journal, vol. 4, no. 4, pp. 99-105, 2015.

[16] A. Addisu, Y. Tegegne, Y. Mihiret, A. Setegn, and A. J. Zeleke, "A 7-year trend of malaria at primary health facilities in Northwest Ethiopia," Journal of Parasitology Research, vol. 2020, Article ID 4204987, 5 pages, 2020.

[17] H. Tesfa, A. G. Bayih, and A. J. Zeleke, "A 17-year trend analysis of malaria at Adi Arkay, North Gondar zone, Northwest Ethiopia," Malaria Journal, vol. 17, no. 1, pp. 155-156, 2018.

[18] A. Alelign, Z. Tekeste, and B. Petros, "Prevalence of malaria in Woreta town, Amhara region, Northwest Ethiopia over eight years," BMC Public Health, vol. 18, no. 1, pp. 1-6, 2018.

[19] A. Alemu, D. Muluye, M. Mihret, M. Adugna, and M. Gebeyaw, "Ten year trend analysis of malaria prevalence in Kola Diba, North Gondar, Northwest Ethiopia," Parasites \& vectors, vol. 5, no. 1, pp. 1-6, 2012.

[20] S. Ergete, S. Sorsa, E. Loha, and S. Asnake, "Trend of malaria cases in Hana and Keyafer health centers, south Omo zone, southern Ethiopia," Ethiopian Journal of Health Sciences, vol. 28, no. 3, pp. 277-286, 2018.

[21] D. G. Feleke, D. Gebretsadik, and A. Gebreweld, "Analysis of the trend of malaria prevalence in Ataye, North Shoa, Ethiopia between 2013 and 2017," Malaria Journal, vol. 17, no. 1, pp. 16, 2018.

[22] M. Yimer, T. Hailu, W. Mulu, B. Abera, and W. Ayalew, “A 5 year trend analysis of malaria prevalence with in the catchment areas of Felegehiwot referral hospital, Bahir Dar city, Northwest-Ethiopia: a retrospective study," BMC Research Notes, vol. 10, no. 1, pp. 1-4, 2017.

[23] D. Gebretsadik, D. G. Feleke, and M. Fiseha, "Eight-year trend analysis of malaria prevalence in Kombolcha, South Wollo, 
north-central Ethiopia: a retrospective study," Parasites \& Vectors, vol. 11, no. 1, pp. 1-6, 2018.

[24] D. Haile, A. Ferede, B. Kassie, A. Abebaw, and Y. Million, "Five-year trend analysis of malaria prevalence in Dembecha Health Center, West Gojjam Zone, Northwest Ethiopia: a retrospective study," Journal of Parasitology Research, vol. 2020, Article ID 8828670, 7 pages, 2020.

[25] B. Berhe, F. Mardu, H. Legese, and H. Negash, "Seasonal distribution and seven year trend of malaria in North West Tigrai: 2012-2018, Ethiopia; 2019," Tropical diseases, travel medicine and vaccines, vol. 5, no. 1, pp. 1-7, 2019.

[26] A. Minwuyelet, T. Eshetu, D. Milikit, and Y. Aschale, "Prevalence and Risk Factors of Asymptomatic Plasmodium Infection in Gondar Zuria District, Northwest Ethiopia," Infection and Drug Resistance, vol. 13, pp. 3969-3975, 2020.

[27] F. Yimer, A. Animut, B. Erko, and H. Mamo, "Past five-year trend, current prevalence and household knowledge, attitude and practice of malaria in Abeshge, south-central Ethiopia," Malaria Journal, vol. 14, no. 1, p. 230, 2015.

[28] A. Abate, A. Degarege, and B. Erko, "Community knowledge, attitude and practice about malaria in a low endemic setting of Shewa Robit Town, northeastern Ethiopia," BMC Public Health, vol. 13, no. 1, p. 312, 2013.

[29] FMOH, "An epidemiological profile of malaria in Ethiopia," in National Malaria Control Team \& Ethiopian Public Health Institute, Federal Ministry of Health, Addis Ababa, Ethiopia, 2014.

[30] USA, Ethioipia, "President's Malaria Initiative EthiopiaMalaria Operational Plan FY 2019,” 2019.

[31] M. Fekadu, M. K. Yenit, and A. M. Lakew, "The prevalence of asymptomatic malaria parasitemia and associated factors among adults in Dembia district, Northwest Ethiopia, 2017," Archives of Public Health, vol. 76, no. 1, pp. 1-6, 2018.

[32] T. Deress and M. Girma, "Plasmodium falciparum and Plasmodium vivax prevalence in Ethiopia: a systematic review and meta-analysis," Malaria research and treatment, vol. 2019, Article ID 7065064, 12 pages, 2019.

[33] T. M. Abdallah, M. T. Abdeen, I. S. Ahmed, H. Z. Hamdan, M. Magzoub, and I. Adam, "Severe Plasmodium falciparum and Plasmodium vivax malaria among adults at Kassala Hospital, eastern Sudan," Malaria Journal, vol. 12, no. 1, pp. 1-7, 2013.

[34] J. Beeson and G. Brown, "Pathogenesis of Plasmodium falciparum malaria: the roles of parasite adhesion and antigenic variation," Cellular and Molecular Life Sciences CMLS, vol. 59, no. 2, pp. 258-271, 2002.

[35] A. S. Paul, E. S. Egan, and M. T. Duraisingh, "Host-parasite interactions that guide red blood cell invasion by malaria parasites," Current Opinion in Hematology, vol. 22, no. 3, pp. 220226, 2015.

[36] Y. Aschale, A. Bitew, B. Kassie, A. Mengist, and A. Talie, "Prevalence of malaria and associated risk factors among asymptomatic migrant laborers in West Armachiho District, Northwest Ethiopia," Research and Reports in Tropical Medicin, vol. 9, pp. 95-101, 2018.

[37] A. Alelign and T. Dejene, "Current status of malaria in Ethiopia: evaluation of the burden, factors for transmission and prevention methods," Acta Parasitologica Globalis, vol. 7, no. 1, pp. 1-6, 2016.
[38] W. R. Mukabana, W. Takken, G. F. Killeen, and B. G. J. Knols, "Weather-based prediction of Plasmodium falciparum malaria in epidemic-prone regions of Ethiopia I. Patterns of lagged weather effects reflect biological mechanisms," Malaria Journal, vol. 3, no. 1, pp. 1-11, 2004.

[39] A. J. McMichael, "The health impacts of global climate change: grappling with scenarios, predictive models, and multiple uncertainties," Ecosystem Health, vol. 1, pp. 23-33, 1995. 